\title{
MOLECULAR ULTRATHIN LAVERS AND ORDERED SYSTEMS
}

\begin{abstract}
Monomolecular assemblies on substrates, now termed Langmuir-Blodgett (LB) films, exhibit many interesting properties and can perform functions which give them perspectives to impact advanced technologies and molecular electronics in an important way. In this article we briefly present our recent efforts aimed at the study of nanoelectric phenomena in the Langmuir monolayer formed on a water surface as well as electrochemical properties of Langmuir-Blodgett films deposited on the surface of an ultramicroelectrode involved in microsensors.
\end{abstract}

\section{Introduction}

Information processing technology currently uses inorganic semiconductor structures. Such structures are fabricated in an essentially planar array on the surface of silicon or gallium arsenide, and have been progressively smaller, primarily by advances in lithography. However, the cost of each successive reduction now increases so much that a practical limit may be approaching. In addition, there are severe physical limits which hinder further progress in miniaturisation. An alternative approach is to start with molecules as basic building blocks, and to assemble the molecules together in a controlled manner so as to create a structure with designed electronic function.

This paper describes a design of systems assembled by the Langmuir-Blodgett (LB) technique. The origin of the present interest in LB films can be traced back from the early 60 s, to the works of H. Kuhn [1] who recognized the utility of LB technique as a tool to assemble molecules in a systematic and well-defined manner. The LB technology is based on two successive steps: formation of an organic monomolecular layer of amphiphilic molecules on the surface of water, and transfer of the monolayer onto the solid substrate by its traversal through the air/water interface (Fig. 1). The monolayer is literally a two-dimensional system built of ordered molecules. The preferential orientation of the molecules with a permanent electric dipole results in spontaneous polarization in the monolayer. The conformational and/or orientational changes of the monolayer constituting molecules induced by external stimuli (illumination, chemical reactions, surface pressure) can be monitored by measuring Maxwell's displacement current [2]. The capability of LB technique can be demonstrated by the production of high sensitive electrochemical devices. Their improvement has been introduced by modification of electrodes, e.g. coating the surface with an organic film. Several deposition techniques have been exploited. We tested a new approach in electrode modification: the LB method was applied for this purpose. Significantly enhanced sensitivity of the determination of metal ions in the water solution is demonstrated by the calibration curves obtained for both the bare and the coated ultramicroelectrodes.

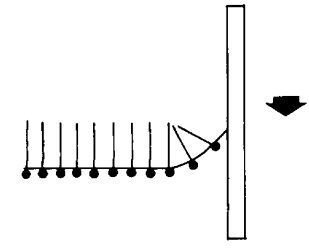

a

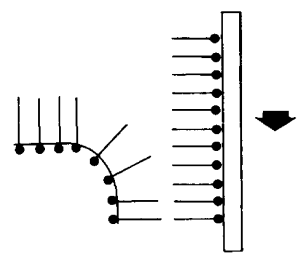

c

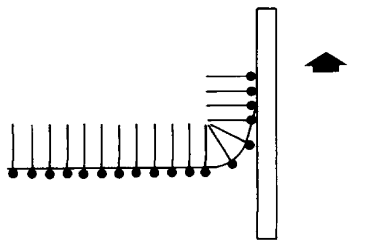

b

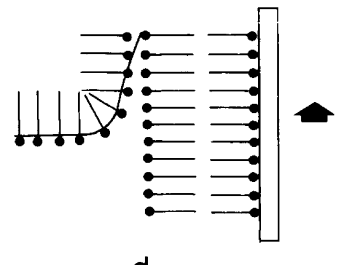

d
Fig. 1

\section{Nanoelectric phenomena in Langmuir monolayer}

We introduced a modified version in which the top electrical contact is detached from the upper surface and the Maxwell displacement current (MDC) is detected in the metal/air gap/Langmuir monolayer/metal structure. MDC flows when the vertical component of the polar molecule changes with time. For this reason, the MDC measuring system is suitable for detecting dielectric relaxation phenomena in a monolayer at the air/water interface. We discuss the evaluation procedure of the molecular dipole moment and some remarks are devoted to the dielectric ralaxation phenomena accompanying the orientational ordering and disordering of monolayers by applying lateral monolayer compression.

Fig. 2 shows the experimental setup in this investigation [3]. The top electrode - TE - (the area is $S=20 \mathrm{~cm}^{2}$ ) was suspended in air, parallel to the water surface. The air gap between $T E$ and the surface was adjusted to $d=0.5 \mathrm{~mm}$ with the aid of a micrometer by monitoring the capacitance of the system. The displacement current was detected by a Keithey 517 electrometer (A). The sensitivity of measuring a current was $0.1 \mathrm{fA}$. The measuring system

\footnotetext{
* Drahoslav Barančok, Július Cirák, Pavol Tomčík, Marek Vančo

Department of Physics, Faculty of Electrical Engineering and Information Technology, Slovak University of Technology, Ilkovičova 3, 81219 Bratislava, E-mail: barancok@elf.stuba.sk, cirak@elf.stuba.sk, tomcik@elf.stuba.sk, vanco@elf.stuba.sk
} 
was attached to the computer-controlled Langmuir trough (NIMA Technology, UK) and placed in a dust-free compartment on an antivibrating block.

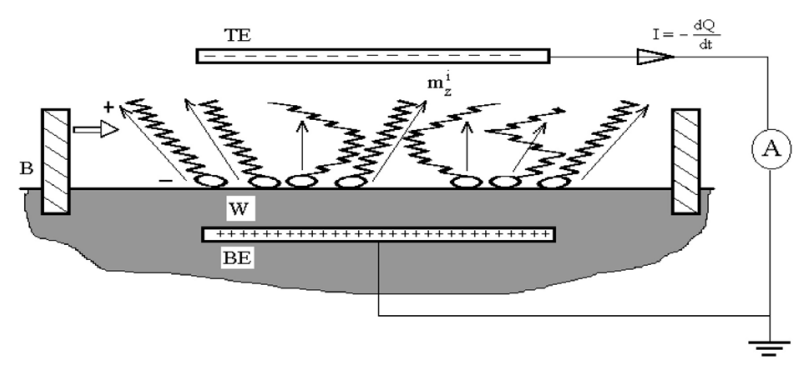

Fig. 2

As a surface-active substance, straight-chain hydrocarbon stearic acid (SA) was used, purchased from Lachema (Brno, Czech Republic). Stearic acid was dissolved in chloroform (1 mmol/1 solution) and slowly added to the surface of bidistilled water to form a single monolayer at the air/water interface. The Langmuir trough was of a rectangular shape with the total working area of $600 \mathrm{~cm}^{2}$. The compression rates varied between 20 and $120 \mathrm{~cm}^{2} / \mathrm{min}$, which corresponded to about 0.05 to $0.30 \mathrm{~A} / \mathrm{s}$ per a molecule.

The displacement current in the circuit originates from three components [4]:

$$
I=\frac{N}{d} \frac{d M}{d t}+\frac{M}{d} \frac{d N}{d t}+\frac{\epsilon_{0} S}{d} \frac{d \Phi}{d t}
$$

The first contribution arises from the change in the vertical component of the molecular dipole moment $(M)$, the second contribution is connected with the change in number of molecules $(N)$ between the electrodes. The third component was taken as zero because the surface potential of water $\Phi$ may be considered constant during the monolayer compression.

A typical recording of the Maxwell displacement current detected simultaneously with the surface pressure $(\pi)$ - area per molecule (A) isotherm during the lateral compression of the monolayer is presented in Fig. 3. The current maximum indicates that the molecules become aligned perpendicularly to the interface. The area under $I-t$ dependence can be utilized for calculation of the change in the induced charge on electrode 1 and, hence, for the calculation of the molecular dipole moment. The value $2.5 \times 10^{-30} \mathrm{Cm}$ or $0.75 \mathrm{D}$ was found for stearic acid molecule. The Maxwell displacement current measurement has been also applied to detect the vertical component of the dipole moment in a poly (3-alkylthiophene) derivative which is able to give a stable monolayer on the water surface. The value $5.5 \times 10^{-30} \mathrm{Cm}$ (or $1.65 \mathrm{D}$ ) are in good agreement with the value obtained from the molecular mechanics calculation [5].

The external compression of the monolayer on a water surface with an aid of a movable barrier can be used for studying non-equi- librium phenomena. For this purpose, the motion of the barrier was not continuous but regularly interrupted so as to allow the monolayer to reach an equilibrium state after stopping the barrier. The transient process was monitored by measuring the displacement current across the monolayer. Experiment showed that the dielectric relaxation time, i.e. the period needed for the monolayer to acquire a new equilibrium state, depends on the monolayer area A and hence on the molecular orientational order. At the beginning of the compression (low orientational order) the transient process is relatively slow $(\tau \sim 3 \mathrm{~s})$. On the other hand, in the state when the molecules stand upright at the air/water interface responds to external compression stimulation much faster $(\tau=1 \mathrm{~s})$. This observation can be supported by the theoretical consideration based on the Debye theory for studying the rotational Brownian motion of molecules with permanent electric dipoles [6].
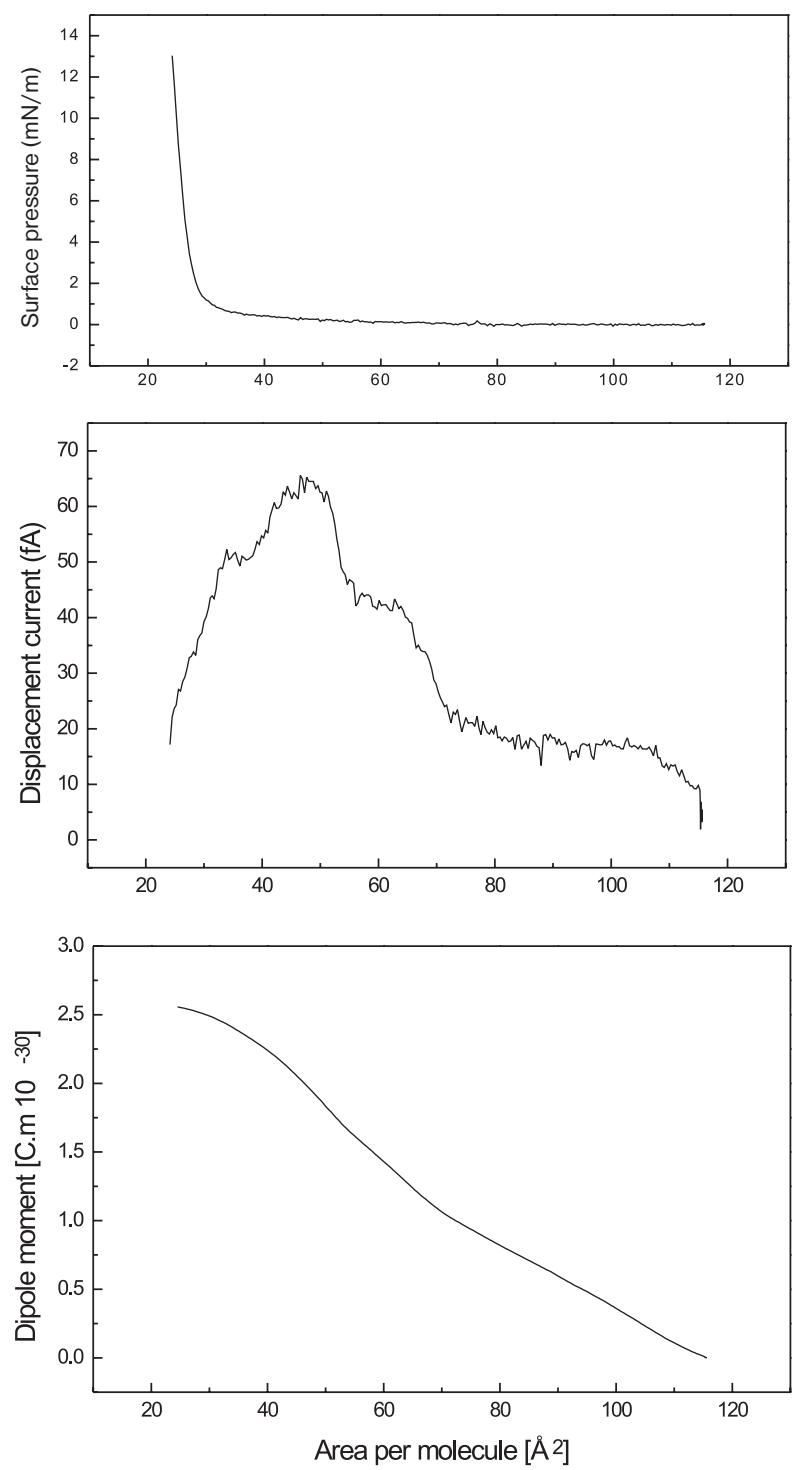

Fig. 2 


\section{Molecular layer as a surface modifier}

The improvement of sensitivity and selectivity of the electrochemical detection system has been introduced by modification of the electrode surface, i.e. coating the surface with thin film for the purpose of immobilizing a specific redox reagent. The superior property of the LB coating method is the capability of depositing a defined number of monolayers (layer-by-layer) of the modifier at ambient temperature, i.e. even a monomolecular-layer coverage is feasible. Substituted polythiophenes were used in the role for the electrode coating, namely amphiphilic poly (3-alkylthiophenes), whose structure (see Fig. 4b) is adjusted to the requirements of the LB process.

The mechanism of charge transport in the layer of a modifier is crucial for kinetics of the charge transfer between the electrode surface and the solution probed. Electrochemical behaviour of both untreated and coated working electrodes was tested by means of double-step voltcoulometry (DSVCM). In this method, the time dependence of the charge transient in response to a potential step is studied at elevating potential applied to electrodes. The kinetic sensitive DSVCM has been presented recently [7]. All the electrochemical experiments were conducted in a two-electrode cell. The carbon fibre working electrode comprised two or three fibres (each $7 \mu \mathrm{m}$ in diameter) protruding from a wax-filled capillary (see Fig. 4a). $\mathrm{An} \mathrm{Ag} / \mathrm{AgCl} /$ electrode was used as a reference. Standard solutions of $\mathrm{Cu}$ were used in testing the working electrode.

(a)

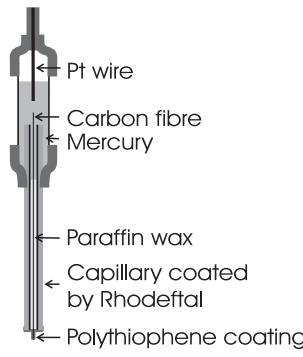

(b)

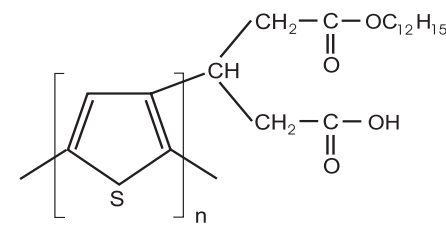

g. 4

The detection ability of the carbon electrode is documented in Fig. 5 where the signal maximum from $\mathrm{Cu}$ ions situated at ca. $-0.3 \mathrm{~V}$ is dependent on the concentration of the species in the solution probed. On the other hand, the signal maximum was considerably raised by the formation of the electrode coating with polythiophene (Fig. 6). The presented potential region $U$ is restricted to the interval, which is dominated by the presence of ions in the electrode redox reaction. Straight calibration lines were observed for a concentration range between 20 and $100 \mathrm{ng} / \mathrm{ml}$ (with a detection limit of $5 \mathrm{ng} / \mathrm{ml}$ ) as shown in Fig.7. The slope of the calibration lines which refers to the sensitivity increases with the number of polymer monolayers and reaches its limit at $10-12$ layers (thickness of the coating being ca. $18 \mathrm{~nm}$ ). The detection process is reversible and the electrode coated by polymer does not "remember" the situation from the previous solution.
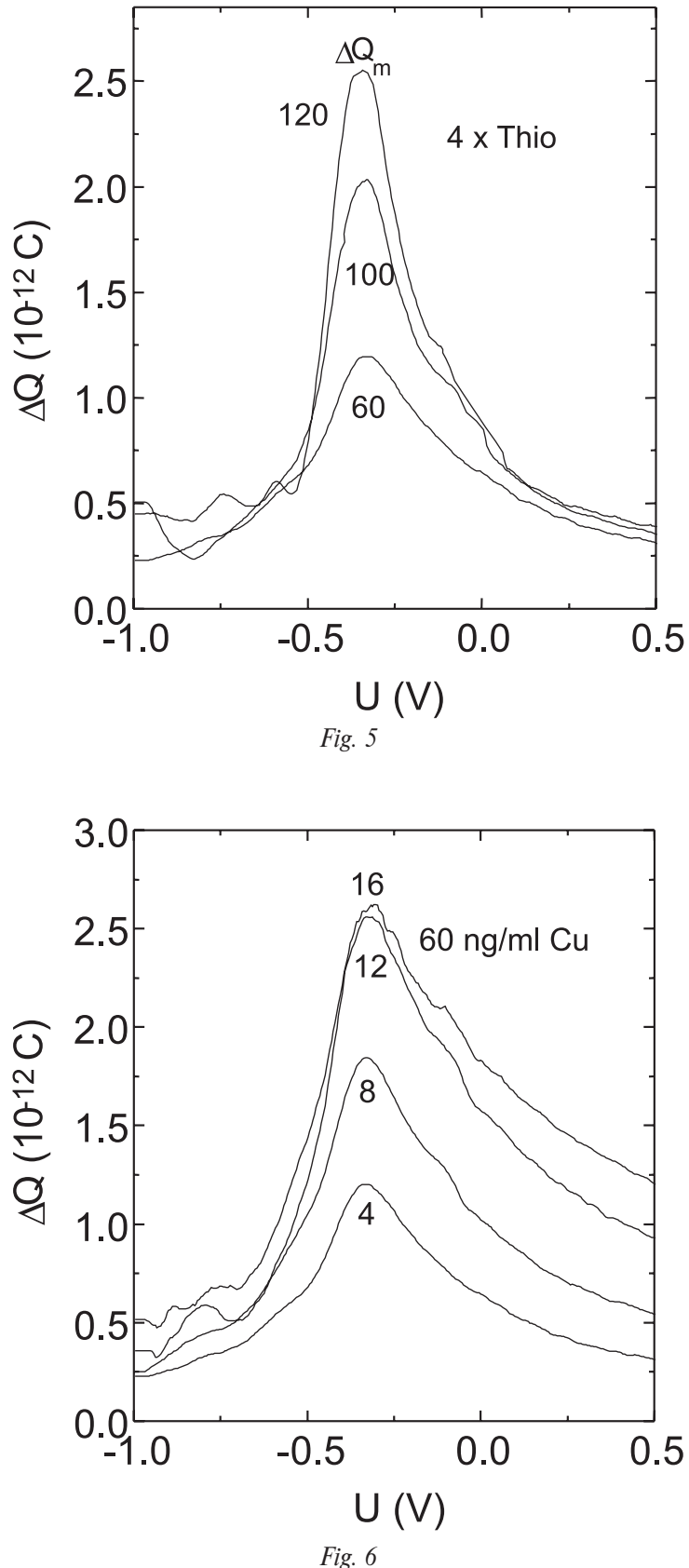

\section{Conclusions}

When defining the role of LB layers in materials science in the new decade and beyond, not only should potential applications be considered but, first of all, the understanding of the molecular assembly in two dimensions at the molecular level is the basis in the technique of materials. Interest is given to the system composed of several types of molecules where the molecular interactions play a key role in determining the resultant system properties. But the interactions depend on the way how the molecules are spontaneously organized in the system - depend on self-orga- 


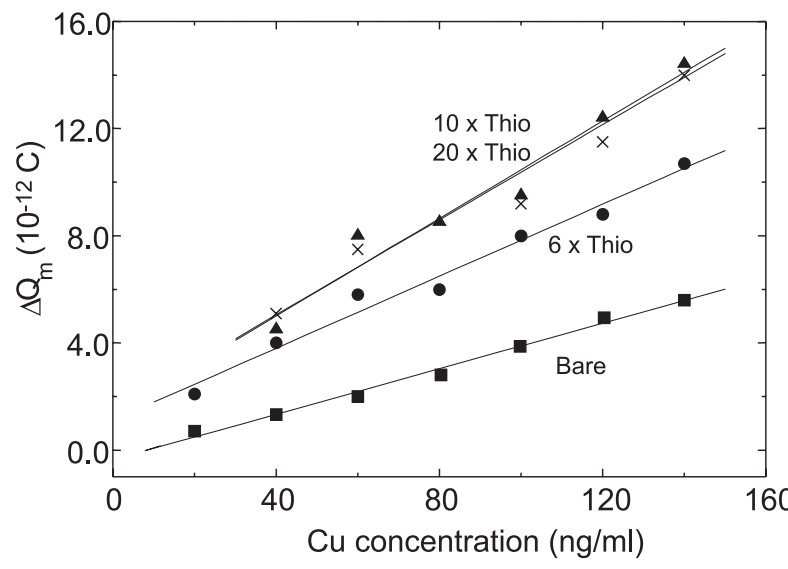

Fig. 7 nization. From this point of view LB films can be regarded as an ideal technological tool for modeling basic physical processes in the system with molecular organization and this impact of the research should be understood and put forward in any concept.

More direct practical goals of the research of organic molecular systems are emerging in these areas: development of electrochemical microsensors, nonlinear optical systems (the use of spontaneous orientation of molecules in the layers, molecular-level control on film thickness, generation of higher harmonics) and development of novel materials for the control of friction and wear (tribology).

\section{References}

[1] KUHN, H.: Naturwissenschaften 54 (1967), 429.

[2] IWAMOTO, M., MAJIMA, Y.,: J. Chem. Phys. 94 (1991), 5135.

[3] BARANČOK, D., CIRÁK, J., TOMČÍK, P., VAJDA, J.: Phys. stat. sol. (a) 169 (1998), 503.

[4] IWAMOTO, M.: Thin Solid Films 244 (1994), 1031.

[5] CIRÁK, J., TOMČÍK, P., BARANČOK, D., BOLOGNESI, A., RAGAZZI, M.: Thin Solid Films 402 (2002), 190.

[6] IWAMOTO, M.: Ch. X. Wu, Phys. Rev. E54 (1996), 6603.

[7] THURZO, I., GMUCOVÁ, K., ORLICKÝ, J., PAVLÁSEK, J.: Rev. Sci. Instrum. 70 (1999), 3723. 